\title{
User rating and acceptance of attention- adaptive driver safety systems
}

\author{
Betina Weber ${ }^{1}$, Manfred Dangelmaier ${ }^{2^{*}}$, Frederik Diederichs ${ }^{2}$ and Dieter Spath ${ }^{1}$
}

\begin{abstract}
Current statistics show that distraction is a central cause of traffic accidents. Safety systems with distance control currently available on the market have great potential for preventing accidents and significantly reducing their severity. However, depending on the driver's level of attention, the systems warn too early or too late, which impairs use acceptance. Adaptive systems allow for personalization according to driver's attention level. Studies were carried out in a driving simulator in order to compare the system adaptations with regard to acceptance for attentive and distracted driving phases. Seventy-two participants took part in the study, with a between-subjects test design. Acceptance ratings shows highest acceptability for the adaptive systems in distractive situations. We conclude that personalization of attention-adaptive systems shall be implemented in case safety benefits are proven.
\end{abstract}

Keywords: System adaptation, Attention level, Safety, Acceptance

\section{Introduction}

Individualization is considered as a megatrend with high societal impact since several years [12]. A vital economy will react on this observation by personalization of products and services provided to the market. The automotive industry fulfils such needs through Mass Customization, creating customized products by means of mass production. The paradigm behind this approach is to provide a customer, who is supposed to be the user of the product, with an appropriate individual solution. During the product configuration, the customer selects among options for several equipment features when ordering a vehicle. Among them are styling features corresponding to personal preferences, but also safety and comfort features like driver assistance and driving automation functions.

However, the presence or absence of features at the time of purchase will not take care of the situational personal needs of users while travelling. In particular, mass customization will do nothing for the changing passengers of a vehicle in a Sharing Economy with a mobility-as-a-

\footnotetext{
* Correspondence: manfred.dangelmaier@iao.fraunhofer.de

${ }^{2}$ Fraunhofer IAO, Institute for Industrial Engineering, Nobelstraße 12, 70569

Stuttgart, Germany

Full list of author information is available at the end of the article
}

service setting. Mass Customization should therefore be changed to something we call Mass Personalization [10, 11]. We define it as: adaptation of mass products/services to invariant as well as changing user needs and to changing users throughout the product lifecycle. Reconfigurable and adaptive systems shall help to achieve these requirements.

This paper deals with the acceptance of attentionadaptive driver safety systems [1]. Either safety systems are provided as standard equipment, usually required by regulations, or customers decide to purchase them as an extra equipment during the configuration of a vehicle in Mass Customization style. Beyond that, attentionadaptive driver safety systems allow for adaptation according to the driver attention during the ride and provide therefore real personalization instead of simple customization. Such systems should not rely on user interaction for adaptation but should be based on sensor data, user models and algorithms for situation handling including artificial intelligence [9]. This avoids negative impact of user preferences on safety. Driver intention recognition allows early warnings, while limiting the frequency of warnings to really relevant situations by algorithms [5]. They can enhance the driver-vehicle 
interaction by offering more intuitive assistance and automated driving support [3].

In 2017 there were 2.6 million accidents in Germany. Three hundred ninety-six thousand six hundred sixty-six injured traffic accident victims were recorded and 3206 seriously injured traffic accident victims have died after accidents [2]. The main cause of all accidents is driver distraction. In order to reduce the number of accidents as well as the number of road deaths and injuries the automotive industry developed Advanced Driver Assistance Systems (ADAS). ADAS and driving automation functions shall avoid accidents caused by distraction. The assistance system or automated functions correct driving errors especially during distraction and in case of emergency. In this way, the system prevents an accident or can reduce the severity of an accident [13].

Conventional, non-adaptive systems tend to warn attentive drivers too early and distracted drivers too late, which leads to complaints and acceptance barriers. This paper investigates an automated Stopping Distance Shortening system (SDS) and compares three systems variants according to acceptability. Two driver state adaptive versions and a non-adaptive version (close to series production). They include collision warning and collision avoidance (autonomous emergency warning and braking).

The study is part of a user centered development process in order to personalize and adapt safety functions in sportive cars. The aim of adapting the systems to the attention level is on the one hand to increase safety in case of distraction and on the other hand to increase acceptance in case of attentive driving.

\section{Methods}

\subsection{Experimental design and system adaptation}

Solving the warning dilemma can be approached in different ways. Solutions might differ in used data related to the driver (e.g. driver skills, fatigue, attention, age), vehicle (e.g. type, speed, acceleration, steering angle, driving maneuver) and environmental conditions (e.g. traffic density, weather conditions). They also might differ in warning strategy parameters such as timing of warnings, intensity of warnings and use of modalities. In this study, the focus is on attention as driver state characteristics. A sports car scenario was chosen for the test. Sports car drivers feel frequently annoyed by false-positives of nonadaptive systems in attentive driving phases. For this reason they may disable the system. This results in a safety risk in critical driving situations. On the other hand, under distraction, earlier warnings and interventions could help to increase safety in critical traffic situations.

Because we were considering longitudinal systems, such as SDS, we were simulating moderate traffic with singular critical traffic events such as a tractor suddenly entering the road in front of the car.

Within a driving simulator study, the system adaptations and automated interventions were tested. Seventy-two test subjects rated the adaptive system functions in a betweensubjects test design. Warning modes and timing of the warnings and interventions were adjusted to three states of attention levels according to the distraction scale BABS $[7,8]$. BABS defines distraction according to several parameters and classifies this secondary task to be highly distractive because it requires one hand and visual attention, typically longer than $2 \mathrm{~s}$ or more than four times in sequence. For this research, three attention levels were tested: highly attentive, normally attentive and distracted. The adaptation of the SDS is described below.

The system goes through different warning and intervention levels. The next level becomes active if the driver does not react to the system warnings. As soon as the driver brakes, the warning and intervention sequence is interrupted.

If the system detects a critical collision situation, the warning level is activated and shows an optical and an acoustic warning that is based on previous work [4] (see Fig. $\left.1,\left(\mathrm{a}, \mathrm{t}_{2}\right)\right)$. If the driver does not react, a brake pulse is

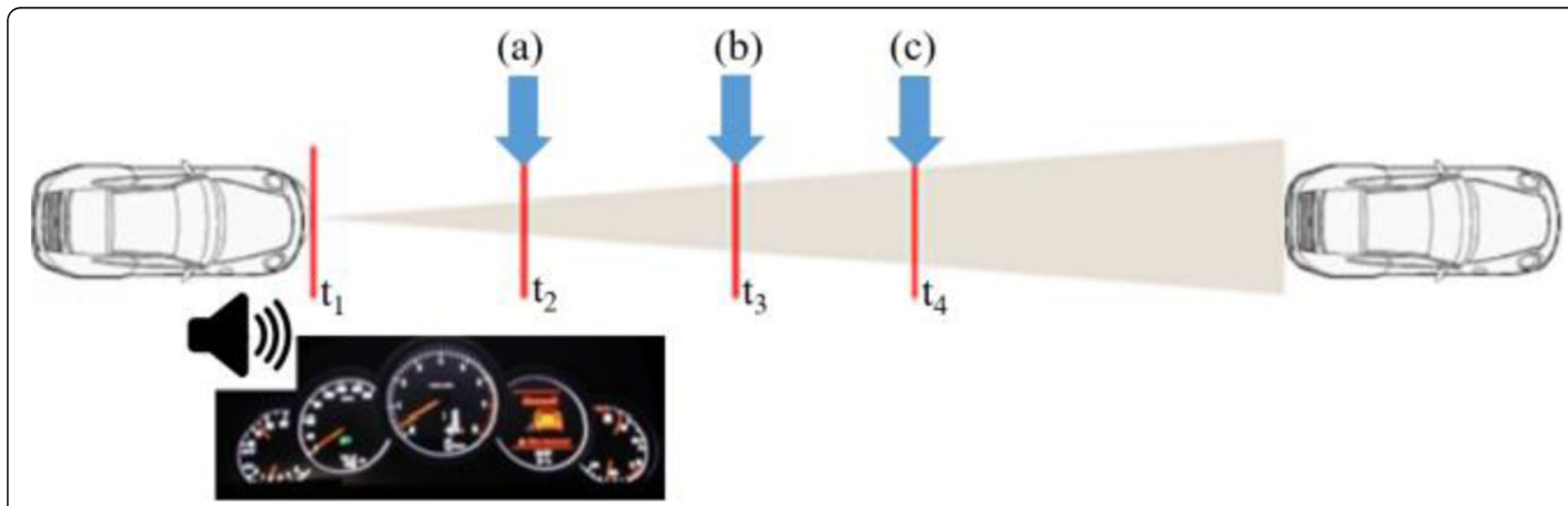

Fig. 1 Non-adaptive, close-to-series system; $\left(\mathbf{a}, \mathrm{t}_{2}\right)$ optical and acoustic warning; $\left(\mathbf{b}, \mathrm{t}_{3}\right)$ brake pulse; $\left(\mathbf{c}, \mathrm{t}_{4}\right)$ partial deceleration 


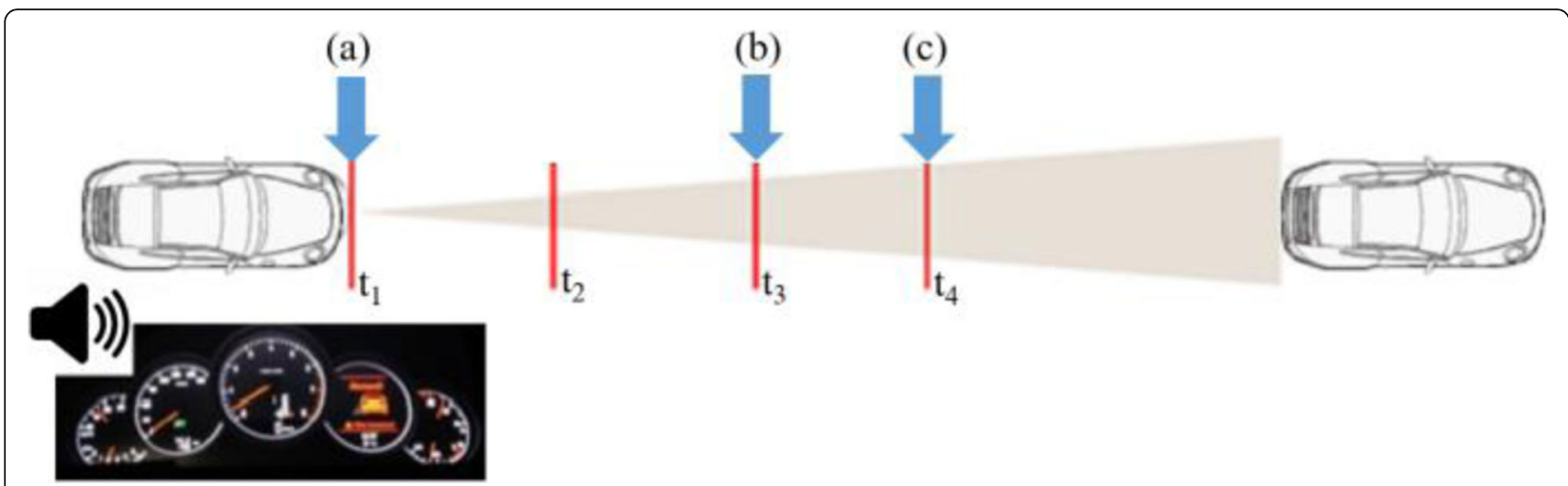

Fig. 2 Distraction mode; $\left(\mathbf{a}, \mathrm{t}_{1}\right)$ optical and acoustic warning; $\left(\mathbf{b}, \mathrm{t}_{3}\right)$ brake pulse; $\left(\mathbf{c}, \mathrm{t}_{4}\right)$ partial deceleration

activated (see Fig. 1, $\left(b, t_{3}\right)$ ) to encourage the driver to brake. If the driver still does not react, an autonomous partial deceleration (see Fig. 1, $\left(\mathrm{c}, \mathrm{t}_{4}\right)$ ) is initiated in order to avoid a collision. The non-adaptive system gives warnings and interventions at fixed, pre-defined time (see Fig. $\left.1, t_{2}, t_{3}, t_{4}\right)$, regardless of the driver's attention level.

This study addressed the problem of the warning dilemma and adapted both the warning and intervention modes as well as the timing of the warnings and interventions on three different attention levels.

Under distraction, the first warning stage occurs earlier (see Fig. $2\left(\mathrm{a}, \mathrm{t}_{1}\right)$ ) for the adaptive systems and the adaptive high-end system ( $4 \mathrm{~s}$ time to collision TTC):

During attentive driving phases the adaptive system shows an optical warning and the adaptive high-end system shows the head up display (HUD) both with a partial deceleration (see Fig. 3 ) at the last possible time ( $t_{4}$,
$1.6 \mathrm{~s}$ time to collision TTC). The acoustic warning as well as the brake pulse is deactivated:

Table 1 shows an overview of the system variants and the warnings and interventions as well as their associated times during attentive driving phases and under distraction. The overview summarizes the system modifications and intervention times for a better understanding of the test settings.

The adaptation of the warning modalities and their timing lead to the following Hypotheses.

\subsection{Hypotheses}

Three hypotheses on the effect of adaptation on user acceptance were investigated.

Hypothesis 1:

In attentive driving phases adaptive SDS will give less or at least the same number of warnings as the conventional

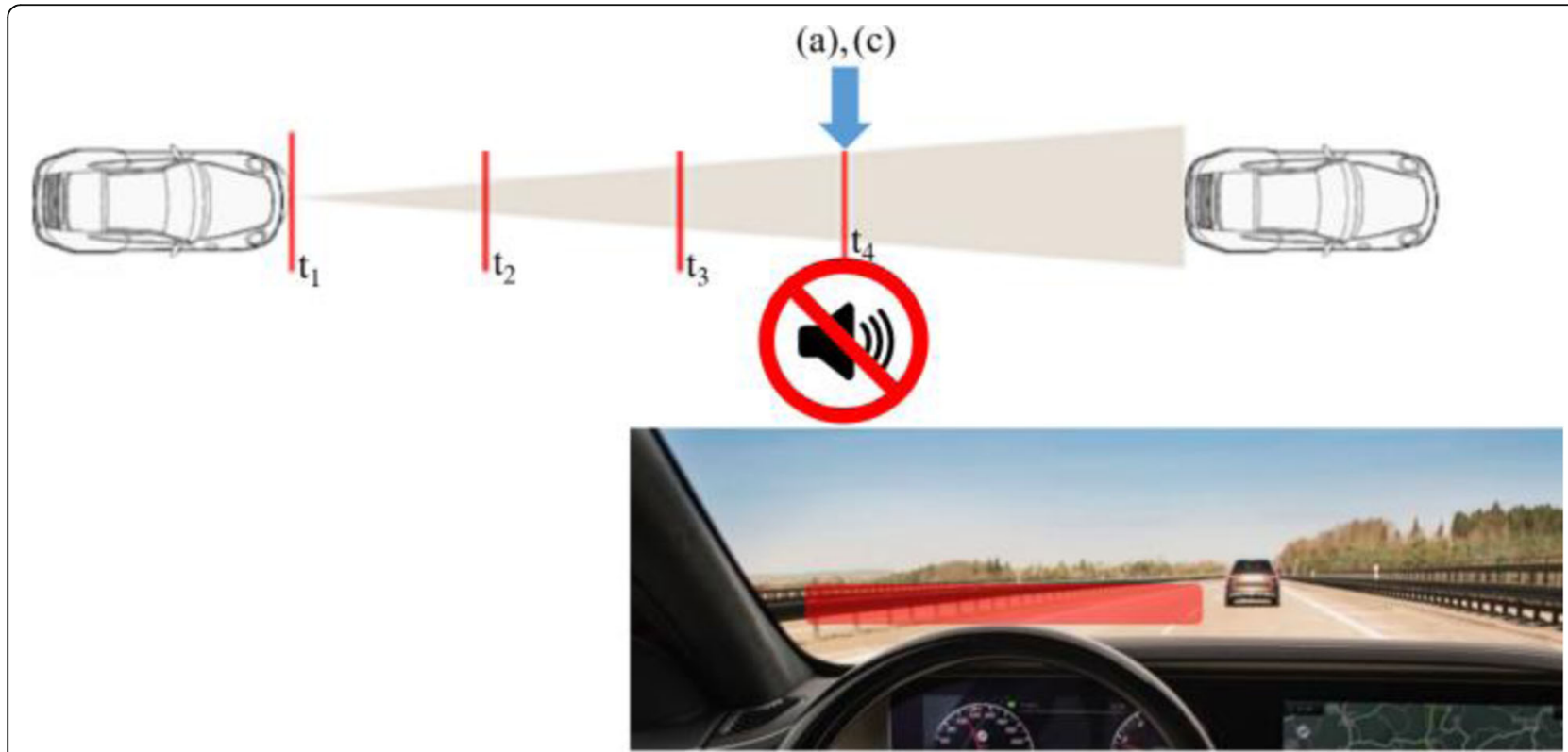

Fig. 3 Highly attentive mode; $\left(\mathbf{a}, t_{4}\right)$ optical HUD warning; $\left(\mathbf{c}, t_{4}\right)$ partial deceleration 
Table 1 Overview of the system variants and the warnings and interventions with their associated times

\begin{tabular}{|c|c|c|c|c|c|c|c|c|c|c|c|c|c|c|c|c|c|c|c|c|c|}
\hline \multirow[b]{3}{*}{ System variant } & \multirow[b]{3}{*}{ Attention level } & \multicolumn{20}{|c|}{ Warnings and interventions } \\
\hline & & \multicolumn{4}{|c|}{$\begin{array}{l}\text { Optical warning } \\
\text { symbol }\end{array}$} & \multicolumn{4}{|c|}{ HUD } & \multicolumn{4}{|c|}{$\begin{array}{l}\text { Acoustic } \\
\text { warning }\end{array}$} & \multicolumn{4}{|c|}{ Brake pulse } & \multicolumn{4}{|c|}{$\begin{array}{c}\text { Partial } \\
\text { deceleration }\end{array}$} \\
\hline & & $\mathrm{t}_{1}$ & $t_{2}$ & $t_{3}$ & $t_{4}$ & $t_{1}$ & $t_{2}$ & $t_{3}$ & $t_{4}$ & $t_{1}$ & $\mathrm{t}_{2}$ & $t_{3}$ & $t_{4}$ & $\mathrm{t}_{1}$ & $t_{2}$ & $t_{3}$ & $t_{4}$ & $t_{1}$ & $t_{2}$ & $t_{3}$ & $t_{4}$ \\
\hline \multirow{2}{*}{$\begin{array}{l}\text { Non-adaptive } \\
\text { system }\end{array}$} & attentive & & $\mathrm{X}$ & & & & & & & & $\mathrm{X}$ & & & & & $\mathrm{X}$ & & & & & $\mathrm{X}$ \\
\hline & distracted & & $\mathrm{X}$ & & & & & & & & $\mathrm{X}$ & & & & & $\mathrm{X}$ & & & & & $\mathrm{X}$ \\
\hline \multirow{2}{*}{$\begin{array}{l}\text { Adaptive } \\
\text { system }\end{array}$} & attentive & & & & $\mathrm{X}$ & & & & & & & & & & & & & & & & $\mathrm{X}$ \\
\hline & distracted & $\mathrm{X}$ & & & & & & & & $\mathrm{X}$ & & & & & & $\mathrm{X}$ & & & & & $\mathrm{X}$ \\
\hline \multirow{2}{*}{$\begin{array}{l}\text { High-end adaptive } \\
\text { system }\end{array}$} & attentive & & & & $\mathrm{X}$ & & & & & & & & & & & & & & & & $\mathrm{X}$ \\
\hline & distracted & & & & & $\mathrm{X}$ & & & & $\mathrm{X}$ & & & & & & $\mathrm{X}$ & & & & & $\mathrm{X}$ \\
\hline
\end{tabular}

SDS, without any compromise on safety. That means Adaptive warning is at least as safe as conventional warning.

Hypothesis 2:

The user acceptance of the adaptive SDS is at least rated as good as the user acceptance of the conventional SDS.

Hypothesis 3:

The adaptive high-end system is better than the adaptive system in terms of global assessment of the system. The adaptive system is better than the conventional system in terms of global assessment of the system.

\subsection{Subjects}

To match the demographic distribution and to cover a broad range of age, two age groups were chosen. One group ranging from 23 to 35 years and another group from 45 to 60 years. In addition, a subdivision was defined by gender (male, female). Conclusively there are four groups of test persons. Eighteen test subjects were scheduled for each group (see Fig. 4), resulting in a number of 72 test subjects in total.

\subsection{Independent variables}

The hypotheses presented in section 2.2 provide information on the safety aspect and system acceptance. The safety aspect is rated and evaluated by an interview after the first test drive. Corresponding CAN data from the driving simulator with respect to the warning and intervention frequencies were logged. The acceptance was rated by a questionnaire after the test drive. Specific questions were selected in order to subsequently evaluate the system variants in a comparison.

\subsection{Driving simulator}

The study was conducted at Fraunhofer IAO in an immersive driving simulator with $180^{\circ}$ front projection and three screens for review mirrors. A one-axis motion base allows braking feedback, which is essential for the scenario design and system experience. The simulator is based on a Renault Scenic (see Fig. 5). It includes a specifically tailored multimodal dashboard with a set of displays behind the steering wheel and in the middle console beside other HMI elements [6].

\subsection{Scenario design}

The test drive is divided into two sections. The first test section was intended for safety purpose. The test drive starts at the end of a village and continues on the country road at $100 \mathrm{~km} / \mathrm{h}$. Oncoming traffic and vehicles
$23-35$ years

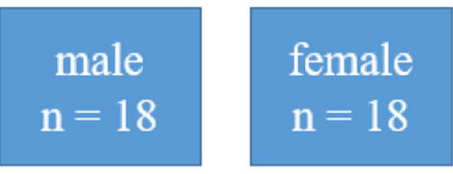

$45-60$ years

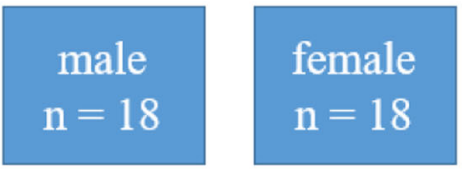

$\mathrm{n}=72$

Fig. 4 Characteristics of the subjects 


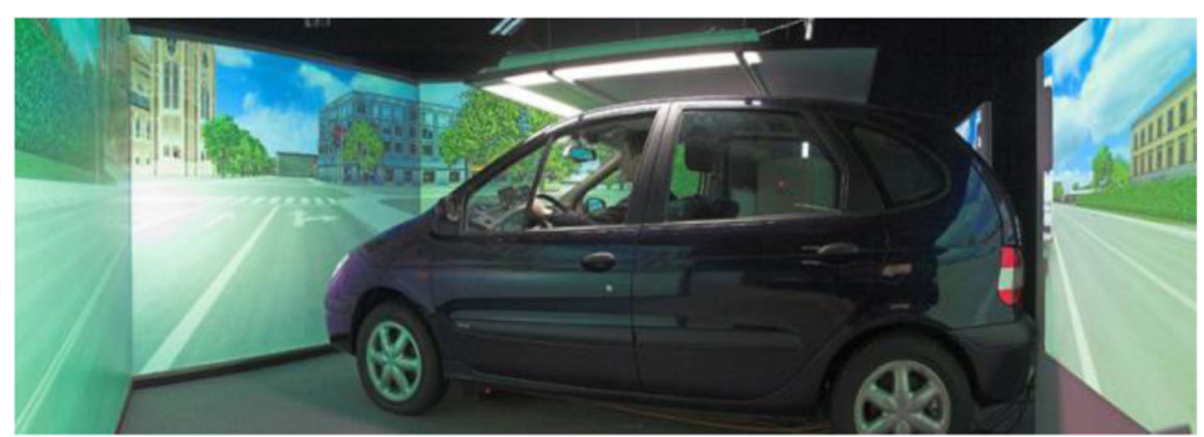

Fig. 5 Immersive driving simulator

waiting at intersections are implemented in the simulation environment to get close to reality. At the end of the scenario, a tractor suddenly appears in front of the vehicle from an intersection to create a critical driving situation to experience the system modalities. 50\% (36 subjects) are attentive and 50\% are distracted in this situation.

The second section is a ride on the highway, in order to compare two system designs with regard to the user acceptance. After prior detailed instruction of the system modalities, the other two out of the three system variants should be directly compared with one another in terms of attention and distraction.

The vehicle drives autonomously. The test subjects can focus on the warning and intervention modes and their timing in near-collision situations. In order to be able to experience them consciously and to be able to evaluate the warnings and interventions, they occur several times in each attention level. After the test drive, both system variants are rated separately for each attention level.

The distraction task had already been validated as a highly distracting counting task in previous studies $[7,8]$. A mixture of red and blue crosses and red circles is shown on a display. The test subject was instructed to count the red crosses within $15 \mathrm{~s}$. A countdown above the symbol arrangement indicates the remaining time [14].

\section{Experimental procedure}

At the beginning, personal data was collected and the test manager gave instructions for the first test phase. The participants started the session by driving a familiarization track. After this familiarization phase participants started a new route. All required functions were explained, except the exact warning modes of the system. At the end of the village the test persons drove directly onto a country road. Likewise the distraction task, which occurred again and again in the center console, should be solved. A speed of $100 \mathrm{~km} / \mathrm{h}$ should be maintained, if possible. Oncoming traffic, occasional rain and waiting vehicles at intersections were also simulated, both with and without distraction, in order to make the test drive as realistic as possible.

At the end of the first driving phase, a tractor suddenly turns in in front of the test person's vehicle. Since CAN data were collected during the test drive, they could be used to compare the warning and intervention frequencies of the system variants.

The second test phase is a comparative drive in which the test persons compared two of the three system characteristics. Each system was experienced both with and without distraction. The rating for system acceptance was realized by questionnaire. A detailed explanation of the forthcoming systems was preceded by the test manager. So it was possible to ensure that the differences in system characteristics and the modalities could be better perceived and evaluated.

\section{Results and discussion}

\subsection{Evaluation of the personal data}

The average age across all test groups is 41 years. The average driving experience is 22.5 years. $20 \%$ of the test persons have less than $5000 \mathrm{~km}$ driving experience per year. $28.6 \%$ of the test persons drive between 5000 and $10,000 \mathrm{~km}$ per year. $30.0 \%$ of the test persons drive between 10,000 and $20,000 \mathrm{~km}$ per year. $21.4 \%$ of the test persons drive more than $20,000 \mathrm{~km}$ per year. $68.1 \%$ of the test persons have no driving simulator experience.

\subsection{Evaluation of the questionnaires}

The global assessment of the adaptive high-end system does not contain any very bad ratings (Fig. 6). 12\% of the test persons rated the system as a whole rather bad, $52.3 \%$ of the test persons rated rather good and $35.7 \%$ of the test persons rated the global assessment of the system very good.

The global assessment of the adaptive system contains $2.6 \%$ very bad ratings. $28.6 \%$ of the test persons rated the system as a whole rather bad, $48.6 \%$ rated rather good and $20.3 \%$ rated the global assessment of the system very good. 
The global assessment of the non-adaptive system contains $4.8 \%$ very bad ratings. $24.9 \%$ of the ratings were ratrher bad for the system as a whole. $47.5 \%$ rated the system rather good and $22.8 \%$ of the test persons rated very good. Two-tailed Wilcoxon test showed no significance at the level $p<0.05$.

The HUD warning of the adaptive high-end system was rated very bad by $4.5 \%$ of the test persons. $11.8 \%$ of the test persons rated rather bad, $16.8 \%$ rated rather good and $66.8 \%$ rated very good.

$18.4 \%$ of the test persons rated the optical warning of the adaptive system very bad. $50 \%$ of the test persons rated rather bad, $18.4 \%$ rated rather good and 13.2\% rated very good.

The optical warning of the non-adaptive system was rated very bad by $34.9 \%$ of the test persons, $23.4 \%$ rated rather bad and $28 \%$ rated rather good. $13.7 \%$ of the test persons rated the optical warning of the non-adaptive system very good. Two-tailed Wilcoxon test showed significance at the level $p<0.05$ with a $p$-value of 0.00398 . Therefore, the result is even significant at $p<0.01$.
The acoustic warning of the adaptive high-end system was rated very bad by $5.1 \%$ of the test persons. $15.5 \%$ of the test persons rated rather bad, $35.5 \%$ of the test persons rated rather good and $43.6 \%$ rated very good.

The acoustic warning of the adaptive system was rated very bad by $3.1 \%, 12.5 \%$ rated rather bad, $41.3 \%$ rated rather good and $43.1 \%$ of the test persons rated very good.

The acoustic warning of the non-adaptive system was rated very bad by $4.8 \%$ of the test persons. $14 \%$ of the test persons rated rather bad, $40.6 \%$ rated rather good and $40.6 \%$ rated very good. Two-tailed Wilcoxon test showed no significance at the level $p<0.05$.

The brake pulse of the adaptive high-end system was rated very bad by $2.4 \%$. $34.1 \%$ rated rather bad, $55.6 \%$ of the test persons rated rather good and $7.9 \%$ rated very good.

The brake pulse of the adaptive system was rated very bad by $5.9 \%$ of the test persons, $22.7 \%$ rated rather bad, $54.4 \%$ rated rather good and $17 \%$ rated the brake pulse of the adaptive system very good.

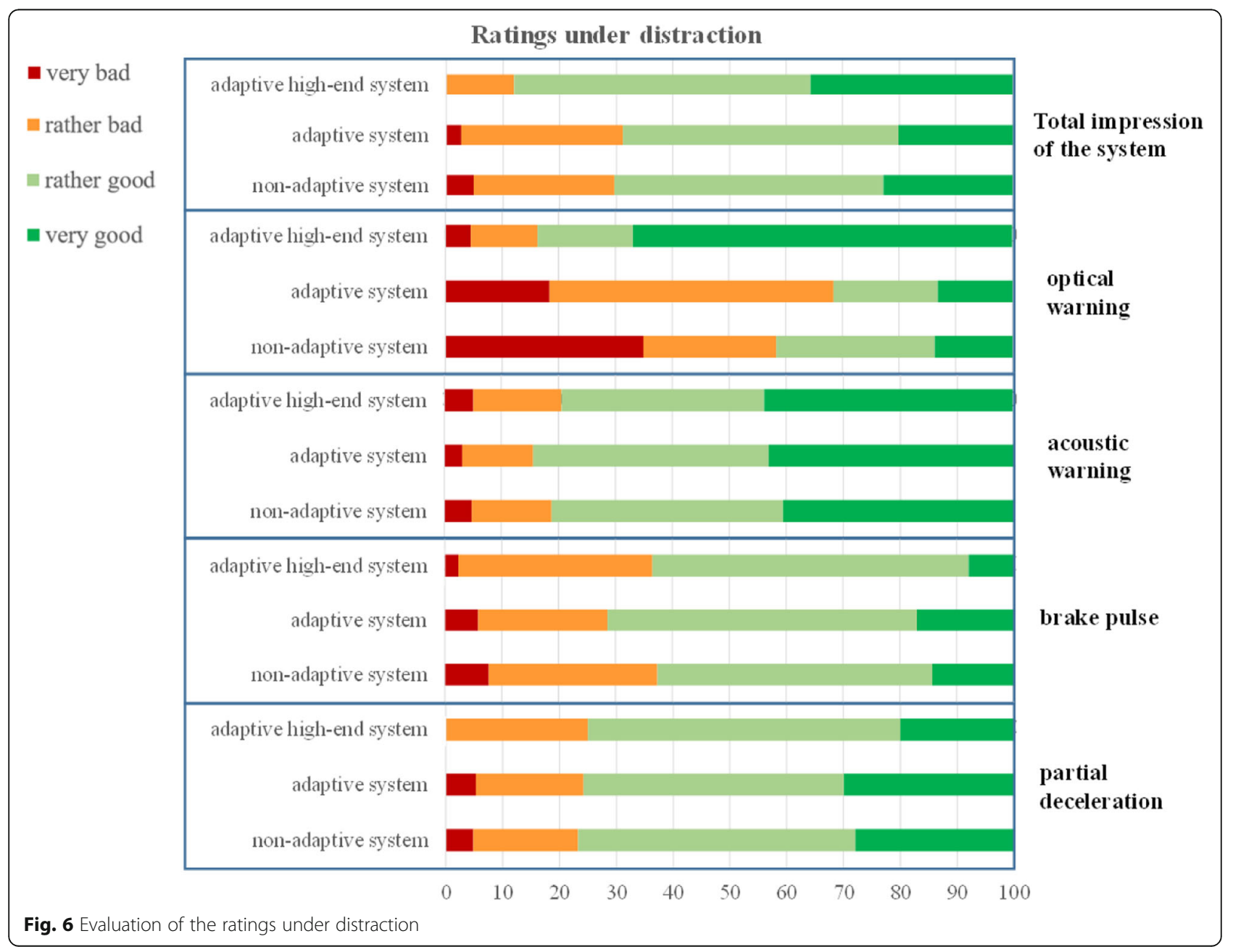


The brake pulse of the non-adaptive system was rated very bad by $7.7 \% .29 .7 \%$ of the test persons rated rather bad, $48.3 \%$ rated rather good and $14.3 \%$ of the test persons rated very good. Two-tailed Wilcoxon test showed no significance at the level $p<0.05$.

The partial deceleration of the adaptive high-end system was rated rather bad by $25 \%$ of the test persons. $50 \%$ of the test persons rated rather, $20 \%$ rated very good. None of the test persons rated very bad.

The partial deceleration of the adaptive system was rated very bad by $5.3 \%$. $18.9 \%$ rated rather bad, $45.9 \%$ rated rather good and $30 \%$ rated the partial deceleration of the adaptive system very good.

The partial deceleration of the non-adaptive system was rated very bad by $4.8 \%$ of the test persons. $18.5 \%$ of the test persons rated rather bad, $48.8 \%$ rated rather good and $27.9 \%$ rated very good. Two-tailed Wilcoxon test showed no significance at the level $p<0.05$.

The global assessment of the adaptive high-end system does not contain any very bad ratings (Fig. 6). 50.9\% of the test persons rated the system as a whole rather bad. $44.7 \%$ rated the rather good and $4.3 \%$ rated the global assessment of the adaptive high-end system very good.

The global assessment of the adaptive system contains $2.1 \%$ very bad ratings. $37.5 \%$ of the test persons rated the system as a whole rather bad, $50 \%$ rated rather good and $10.4 \%$ rated the global assessment of the system very good.
The global assessment of the non-adaptive system does not contain very bad ratings. $25 \%$ of the test persons rated the system as a whole rather bad, $60.4 \%$ rated the system rather good and $14.6 \%$ of the test persons rated very good. Two-tailed Wilcoxon test showed no significance at the level $p<0.05$.

The HUD warning of the adaptive high-end system was rated very bad by $16.7 \% .41 .7 \%$ of the test persons rated rather bad, $22.9 \%$ rated rather good and $18.8 \%$ rated very good.

The optical warning of the adaptive system was rated very bad by $13.1 \%$ of the test persons. $44.5 \%$ rated rather bad, 33.4\% rated rather good and 9\% of the test persons rated very good.

The optical warning of the non-adaptive system was rated very bad by $14.7 \%$. $38.5 \%$ rated rather bad, $29.9 \%$ rated rather good and $16.9 \%$ of the test persons rated very good. Two-tailed Wilcoxon test showed no significance at the level $p<0.05$.

The partial deceleration of the adaptive high-end system was rated very bad by $2.6 \%$ of the test persons. $20.4 \%$ of the test persons rated rather bad. $60.8 \%$ rated rather good and $16.2 \%$ of the test persons rated very good.

$2.2 \%$ of the test persons rated the partial deceleration of the adaptive system very bad. $27.5 \%$ rated rather bad, $56.8 \%$ of the test persons rated rather good and 13.5\% rated very good.

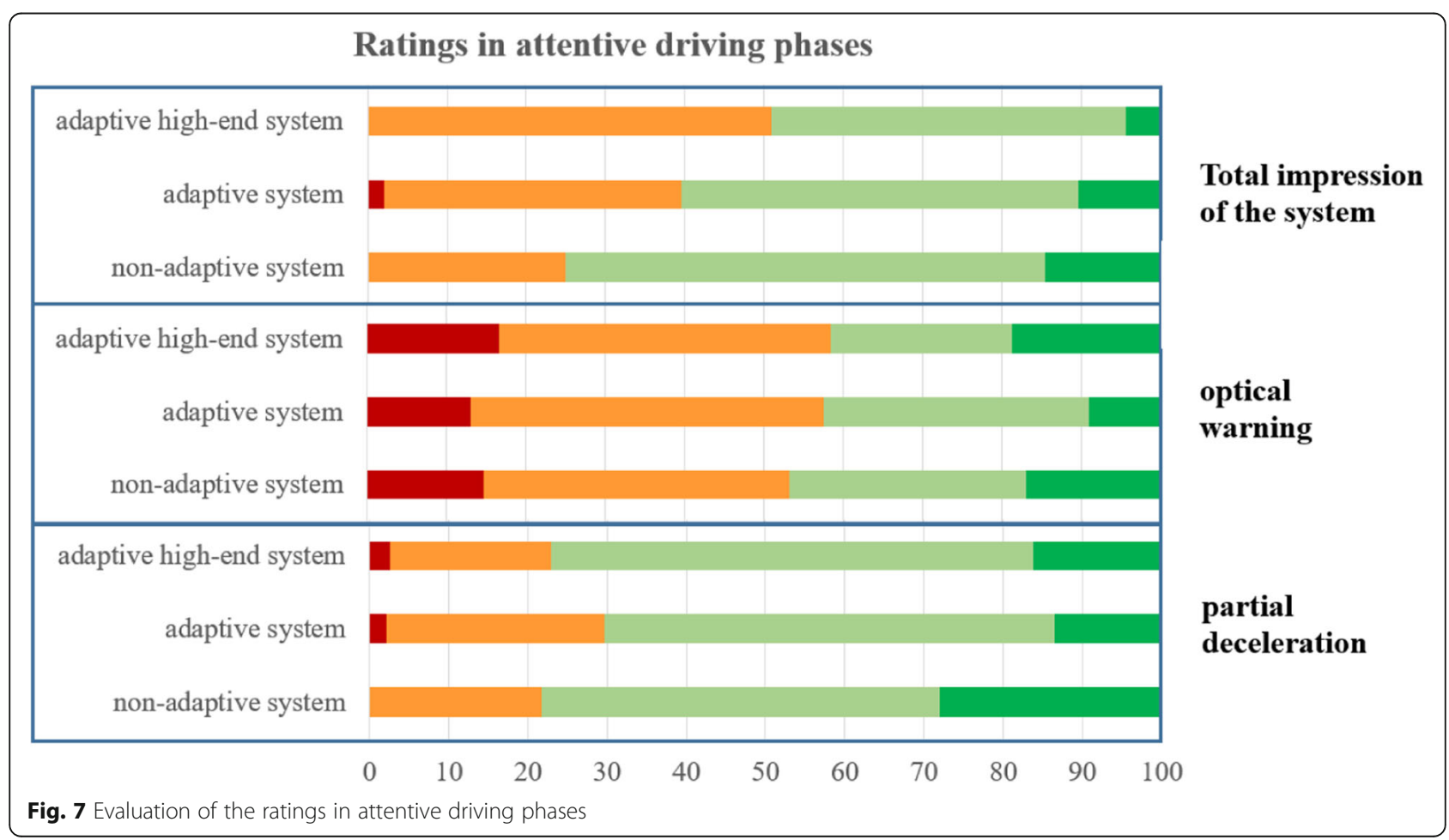




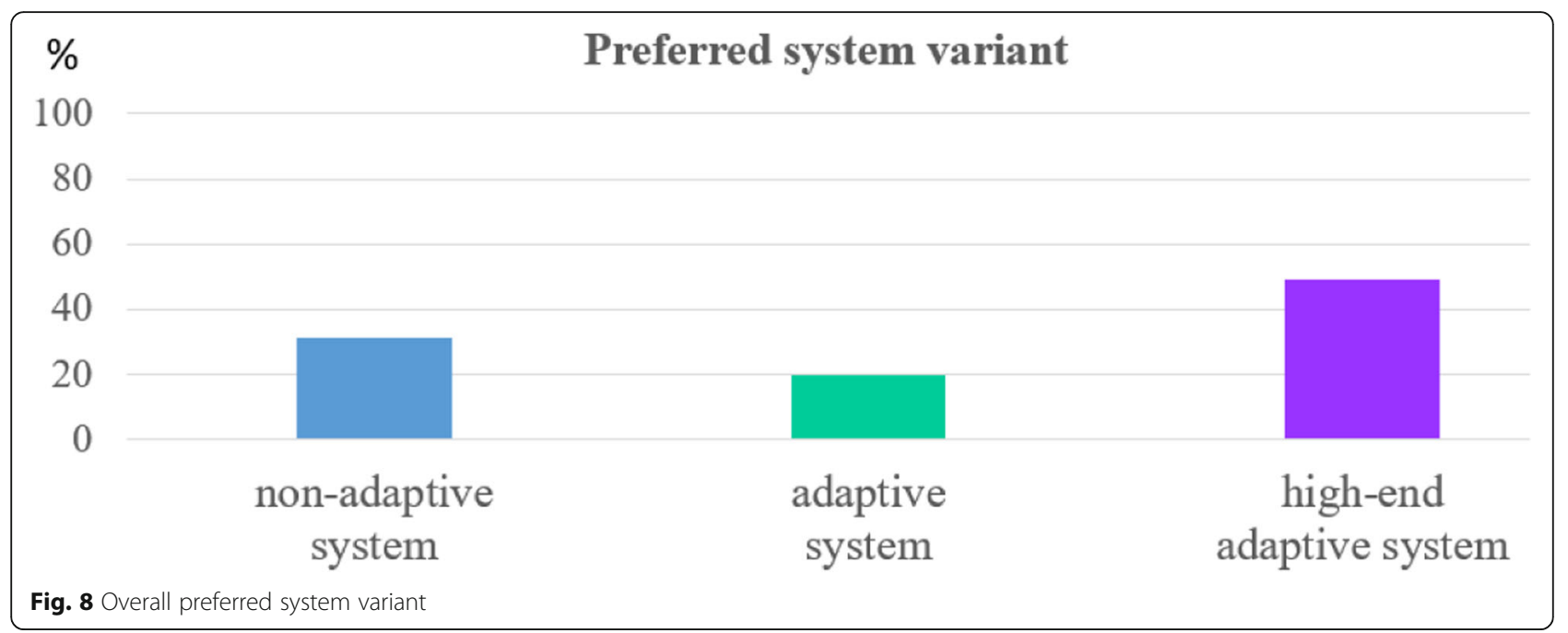

Figure 7 shows the ratings of the total impression, the optical warning and the partial deceleration intervention of the 3 system variants in attentive driving phases.

The partial deceleration of the non-adaptive system was rated rather bad by $21.8 \% .50 .2 \%$ rated rather good and $28 \%$ of the test persons rated very good. None of the test persons rated very bad in any case. Two-tailed Wilcoxon test showed no significance at the level $p<0.05$.

The following figure (Fig. 8) shows the results in direct comparison, which system variant the test persons preferred. Two of the three system variants were shown during the comparison test drive. Each system was seen and evaluated by 48 test persons. In total, the ratings of 72 test persons could be evaluated for three system variants.

The non-adaptive, close-to-series system was preferred by $31 \%$ of the test subjects. The adaptive system was preferred by $19.7 \%$. The adaptive high-end system was preferred by $49.3 \%$ of the test subjects. Two-tailed Wilcoxon test showed no significance at the level $p<0.05$. The difference between the rating of the adaptive system and the high-end adaptive system just missed significance with a $p$-value of 0.0536 .

The following Figs. 9 and 10 show the ratings of warning and intervention times. The attentive driving situation and the test phase under distraction were evaluated seperately. The test persons rating options distingusted between much too early, too early, just right, little too late and much too late. Figure 9 shows the evaluation of the rating for the attentive test drive phase.

Both the adaptive system and the adaptive high-end system was classified by $40 \%$ of the subjects as little too late in attentive driving situations with regard to the

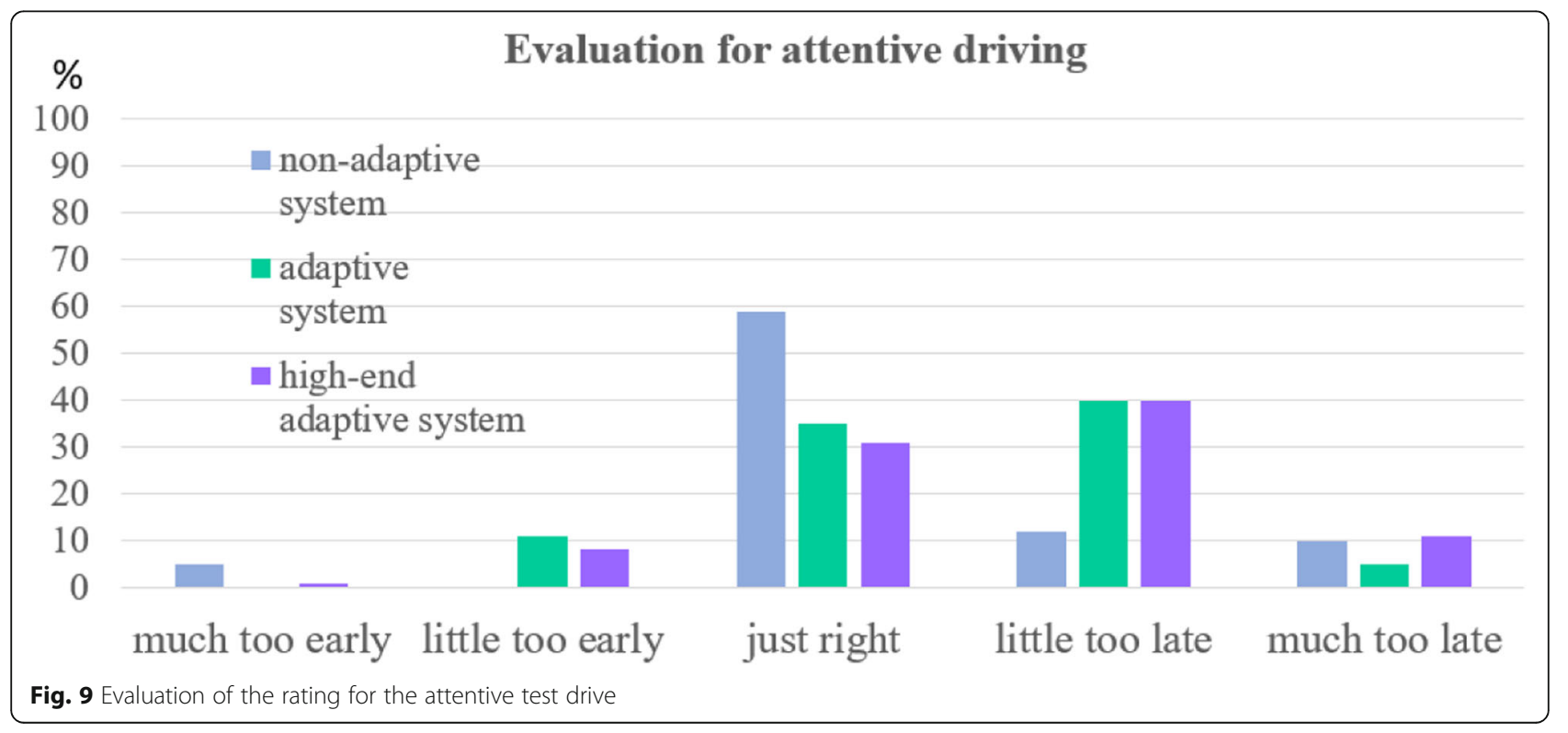




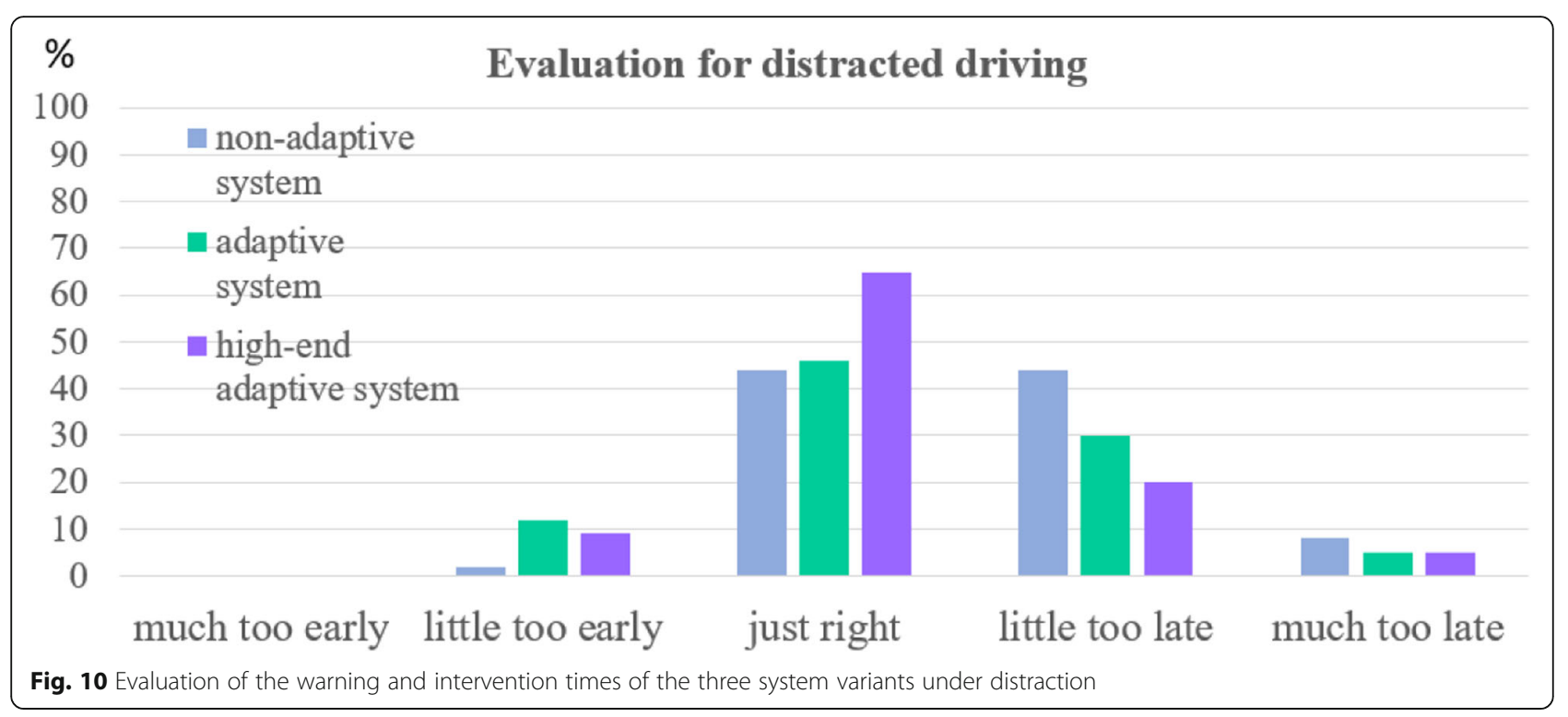

warning and intervention time. The warning and intervention time of the non-adaptive system was rated just right by almost $60 \%$ of the test subjects. The warning and intervention time of the adaptive system and the adaptive high-end system was rated just right by $32 \%$ of the test subjects. Individual ratings were in the areas much too early, little too early and much too late. These are similarly distributed and negligible.

Figure 10 shows the evaluation of the warning and intervention times of the three system variants under distraction.

The warning and intervention times of the adaptive high-end system were rated just right by almost $65 \%$ of the test persons. With about $20 \%$ less in this category, the non-adaptive system and the adaptive system were evaluated. The test drive of the non-adaptive system under distraction was rated most frequently little too late. Followed by the adaptive system with $30 \%$ and $20 \%$ for the adaptive high-end system. For all three system variants the ratings in the categories little too early and much too late are negligible compared to the categories just right and little too late. They are at a similar level. In none of the three system variants the warning and intervention times were rated much too early.

\subsection{Evaluation of the CAN data}

The safety aspect of the SDS system was verified by evaluating and comparing the warning and intervention frequency of all three system characteristics under distraction using CAN data.

The following Fig. 11 shows the results. The vertical axis displays the number of warnings and interventions per system characteristics, for each of the the individual warning modalities.
By far the fewest warnings outputs occur within the nonadaptive system variant. Both adaptive variants show four times more warnings and never go beyond the first warning level. After the visual-acoustic warning, all drivers reacted in time and braked themselves. The non-adaptive system triggered the brake pulse twice. The partial deceleration was activated once. Warnings or interventions were not measured in any of the system variants during attentive driving phases, since the subjects decelerated in time.

\section{Conclusion}

Hypothesis 1 was verified by CAN data recorded during the test drive. Warnings or interventions were not logged in any of the system variants, as all of the test persons braked in time. Both the warning and intervention times of the adaptive system and of the adaptive high-end system in attentive driving phases were classified as little too late by $40 \%$ of the test persons. The earlier warning and intervention times of the non-adaptive system were rated exactly right by nearly $60 \%$ of the test persons. This means that the warnings and interventions of the two adaptive system variants did not interfere. Even the nonadaptive system was accepted, although warnings and interventions occur earlier in attentive driving phases.

With both adaptive system variants occurred four times more warnings under distraction than with the non-adaptive system variant. Nevertheless, the warning and intervention times of the adaptive high-end system were rated exactly right by the majority of the test persons. The adaptive system is rated slightly better than the non-adaptive system variant. This means that the warnings and interventions of the two adaptive system variants did not interfere. Even the non-adaptive system variant, which issues warnings and intervenes during 


\section{Warnings and interventions under distraction}

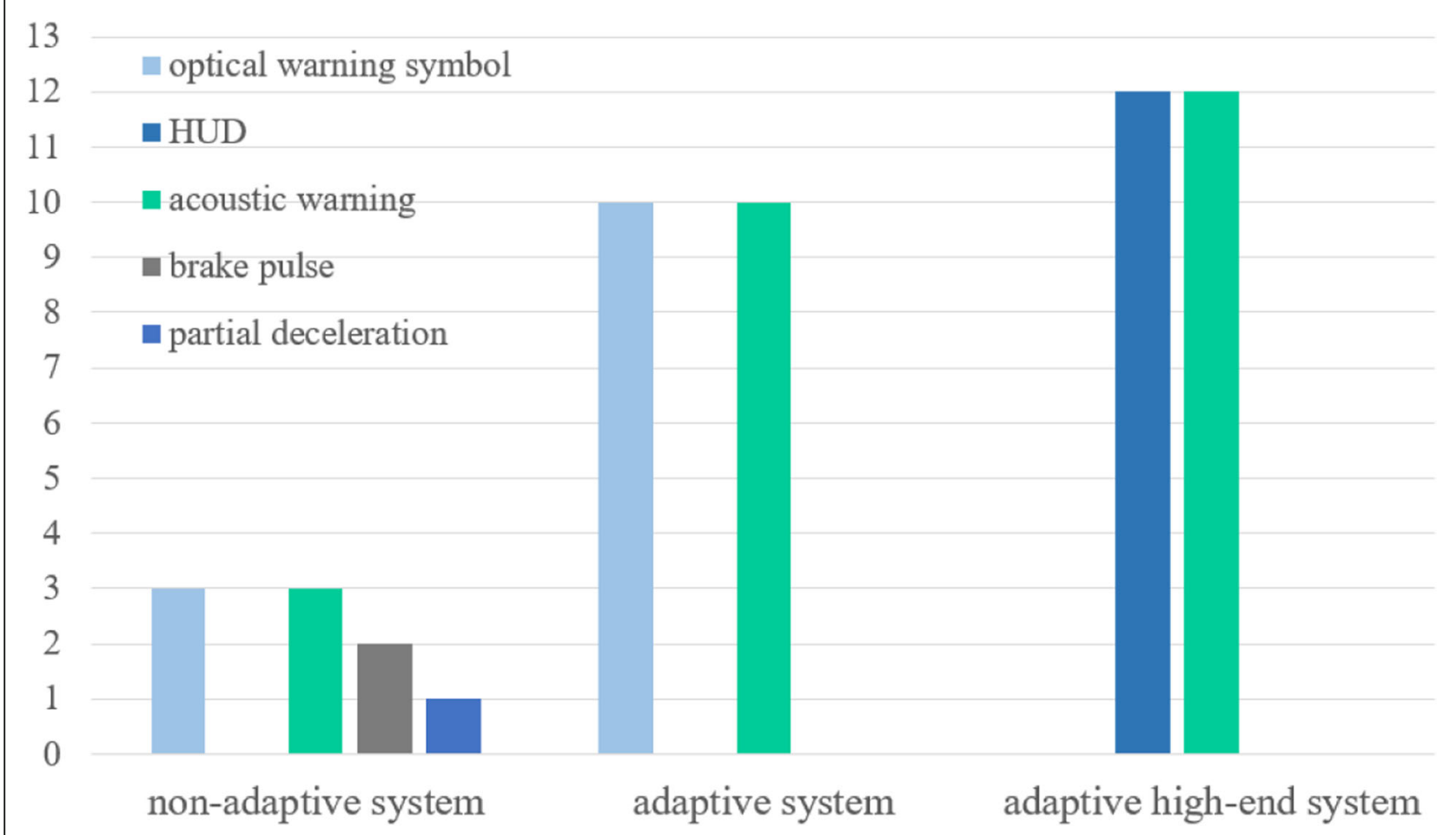

Fig. 11 Evaluation of the warning and intervention frequency under distraction

attentive driving phases, was accepted. As a result, the adjustment of the warning or intervention times has no negative effect on the system ratings.

The change of the warning modality from optical warning symbol to HUD warning bar as a head up display has a clear, positive effect on the rating of time, although all warnings and interventions occur at the same time. The modification of the optical warning modality therefore has a positive effect on the total perception of the system and its evaluation.

Hypothesis 2 was verified by rating the individual warning and intervention modalities as well as the total rating of the system variants. Both in attentive driving phases and under distraction. In addition, the preferred system variant and the preferred variant in the respective attention level were also rated. In attentive driving phases and under distraction the test persons were asked, how the warning and the intervention times were experienced. The system evaluation of the non-adaptive system under distraction is clearly different compared to the adaptive system, although the warning modality did not change. Only the warning and intervention times occurred earlier.

The warning modality optical warning symbol was rated much better for the adaptive system variant than for the non-adaptive system variant, although the same display was implemented. That means that the rating of the warning modality optical warning symbol is significantly improved by an earlier warning under distraction.

In the adaptive system variant, more than $15 \%$ fewer very bad ratings were given for the warning modality optical warning symbol compared to the non-adaptive system variant.

The adaptive high-end system replaces the optical warning symbol by an HUD warning display as a headup display. The difference in the system evaluation becomes here even clearer.

Especially in comparison to the non-adaptive system, but also in comparison to the adaptive system, there is a clear improvement regarding the rating of the system. This can be verified both in the individual warning mode HUD warning display compared to the optical warning symbol as well as in the global assessment of the system. The adaptive high-end system is rated as rather good or very good by more than $80 \%$ of the test persons in the HUD warning, which represents an improvement of more than $20 \%$ compared to the non-adaptive system. This very positive evaluation of the HUD warning also has a significant effect on the evaluation of the system as a whole. Almost $90 \%$ of the test persons rated the global assessment of the adaptive high-end system as rather good or very good. This shows that the optical warning in combination with an earlier warning under distraction 
has a significantly positive effect on the evaluation of the system as a whole.

The other warnings and interventions show hardly any differences and no clear tendencies between the different system variants. The same applies to the attentive test drive. Here are the three system variants and their warning and intervention modalities were rated similarly. Under distraction, the timing of the warnings and interventions of the adaptive high-end system is classified as exactly right by nearly $65 \%$ of the test persons. This is $20 \%$ more than the adaptive system and non-adaptive system. The warning and intervention times of the nonadaptive system are rated as little too late by $45 \%$ of the test persons. During the test attentive test drive, however, the non-adaptive system is rated as just right with a $25-30 \%$ lead over the adaptive system and the adaptive high-end system with its warning and intervention times. Both adaptive system variants are rated as little too late by $40 \%$ of the test persons. Overall, the adaptive highend system was clearly preferred by $50 \%$ of the test persons.

To verify hypothesis 3 , the same questions and associated results were used that were also relevant for hypothesis 2. Although the warning and intervention frequency of the adaptive systems was four times higher compared to the non-adaptive system under distraction, the acceptance under distraction is by far at highest level, especially in the adaptive high-end system variant. The results show that the adaptive high-end system was rated better than the non-adaptive system. The results, which also verify hypotheses 1 and 2 , show that the adaptive high-end system was rated better than the adaptive system and that the adaptive system was rated better or similar to the non-adaptive system.

The results show no significant differences for the global assessments of the systems neither in attentive driving phase nor under distraction, although the warning and intervention frequency of the high-end adaptive system was four times higher compared to the nonadaptive system under distraction. That means that earlier warnings and interventions of the high-end adaptive system do not interfere user acceptance in a negative manner, although increase of safety level is achieved.

\section{Declarations}

The research topic fits in the main areas of interest of the ETRR.

\footnotetext{
Authors' contributions

BW planned the system modifications as well as the simulation scenarios, analyzed and interpreted the data and was a major contributor in writing the manuscript. MD established the context and relation of Mass Customization and Mass Personalization, supported the presentation of the analyzed data and was a major contributor in writing the manuscript. FD supported the planning of the experiments and the design of the questionnaires as well as in the presentation of results. DS established the research program, conceptualized the research questions, supervised the researchers, and advised on methods as well as on quality of work. All
}

authors read and approved the final manuscript for submission. The content of the manuscript has not been published, or submitted for publication elsewhere.

\section{Funding}

Part of the research was conducted for the diploma thesis of the corresponding author at the University of Stuttgart. The work was supported by Porsche AG, by Fraunhofer-Gesellschaft zur Förderung der angewandten Forschung e. V. and by the State of Baden-Württemberg in the context of the Leistungszentrum Mass Personalization.

\section{Availability of data and materials}

The data that support the findings of this study are available from Fraunhofer Institute for Industrial Engineering IAO but restrictions apply to the availability of these data, which were used under license for the current study, and so are not publicly available. Data are however available from the authors upon reasonable request and with permission of Porsche AG.

\section{Competing interests}

The authors declare that they have no competing interests.

\section{Author details}

${ }^{1}$ Universität Stuttgart, Institut für Arbeitswissenschaft und Technologiemanagement, Nobelstraße 12, 70569 Stuttgart, Germany.

${ }^{2}$ Fraunhofer IAO, Institute for Industrial Engineering, Nobelstraße 12, 70569 Stuttgart, Germany.

Received: 9 July 2019 Accepted: 8 March 2020

Published online: 01 May 2020

\section{References}

1. Amditis, A., Polychronopoulos, A., Andreone, L., \& Bekiaris, E. (2006), Communication and interaction strategies in automotive adaptive interfaces. Cognition, Technology \% Work, 8(3), 193-199.

2. Bundesanstalt für Straßenwesen (Ed.), 2018. Infrastruktur-, Verkehrs- und Unfalldaten. In: https:/www.bast.de/BASt_2017/DE/Presse/Mitteilungen/201 7/presse-17-2017.html.

3. Diederichs, F., Brouwer, N., Klöden, H., Zahn, P., \& Schmitz, B. (2018). Application of a Driver Intention Recognition Algorithm on a Pedestrian Intention Recognition and Collision Avoidance System. In UR:BAN Human Factors in Traffic (pp. 267-284). Wiesbaden: Springer Vieweg.

4. Diederichs, F., Marberger, C., \& Hinder, V. (2010). Iterative design and assessment of an audio visual warning concept for Car2x communication systems. In Proceedings of the 17th ITS World Congress on Intelligent Transportation Systems. Busan.

5. Diederichs, F., Schüttke, T., \& Spath, D. (2015). Driver Intention Algorithm for Pedestrian Protection and Automated Emergency Braking Systems. In IEEE 18th International Conference on Intelligent Transportation Systems ITSC (pp. 1049-1054). Canary Islands.

6. Diederichs, F., Weber, B., \& Ganzhorn, M. (2011). Experimental Evaluation of ITST based School Bus Stop Notifications for Car Drivers. In 11th International Conference on ITS Telecommunications. St. Petersburg.

7. Ganzhorn, M., Diederichs, F., \& Spath, D. (2012). Echtzeitmessung zur Erfassung und Beurteilung von Ablenkung im KFZ, 28th VDII VWGemeinschaftstagung Fahrerassistenz und Integrierte Sicherheit. Wolfsburg.

8. Ganzhorn, M., Diederichs, F., \& Höfer, M. (2013). Eine bewerterbasierte Ablenkungsskala (BABS) zur objektiven Beurteilung von Unaufmerksamkeit, 7th VDl-Tagung. Braunschweig.

9. Gillmeier, K., Schüttke, T., Diederichs, F., Miteva, G., \& Spath, D. (2018). Combined driver distraction and intention algorithm for maneuver prediction and collision avoidance. In IEEE International Conference on Vehicular Electronics and Safety. Madrid.

10. Hämmerl, R., \& Dangelmaier, M. (2018). Mass Personalization und die Erfolgsfaktoren nach dem Stuttgarter Modell. ZWF, 113(11), 730-733.

11. Held, M., Wehner, D., Hämmerl, R., Dangelmaier, M., Briem, A.-K., Reiff, C., \& Wulle, F. (2018). Personalization in the automotive and building sector. In 8th International Conference on Mass Customization and Personalization MCP - CE 2018. Novi Sad.

12. Naisbitt, J. (1982). Megatrends: Ten new directions transforming our lives (1st ed.p. 290). New York: Warner Books. 
13. Schroven, F. (2011). Dissertation: Probabilistische Situationsanalyse für eine adaptive automatisierte Fahrzeuglängsführung, Technische Universität CaroloWilhelmina zu Braunschweig. Braunschweig.

14. Weber, B., Höfer, M., Diederichs, F., Dangelmaier, M., \& Spath, D. (2019). Acceptance of attention-adaptive driver assistance systems, Stuttgarter Symposium für Produktentwicklung SSP. Stuttgart.

\section{Publisher's Note}

Springer Nature remains neutral with regard to jurisdictional claims in published maps and institutional affiliations.

\section{Submit your manuscript to a SpringerOpen ${ }^{\circ}$ journal and benefit from:}

- Convenient online submission

- Rigorous peer review

- Open access: articles freely available online

- High visibility within the field

- Retaining the copyright to your article

Submit your next manuscript at $\boldsymbol{\wedge}$ springeropen.com 